\title{
Patients' Adult Attachment Interview classification and their experience of the therapeutic relationship: are they associated?
}

\author{
Alessandro Talia, ${ }^{1}$ Madeleine Miller-Bottome, ${ }^{2}$ Rachel Wyner, ${ }^{3}$ Peter Lilliengren, ${ }^{4}$ Jordan Bate ${ }^{5}$
}

${ }^{1}$ Institute for Psychosocial Prevention, Heidelberg University Hospital, University of Heidelberg, Heidelberg, Germany; ${ }^{2}$ Department of Psychology, The New School for Social Research, New York, USA; ${ }^{3}$ William Alanson White Institute, New York, USA; ${ }^{4}$ Ersta Sköndal Bräcke University College, Stockholm, Sweden; ${ }^{5}$ Ferkauf Graduate School of Psychology, Yeshiva University, New York, USA

\begin{abstract}
In the last decade of his career, Jeremy Safran became increasingly interested in investigating the ways in which attachment representations influence the therapeutic relationship. In this paper, we test such influence in a sample of thirty outpatients who received Brief Relational Therapy by comparing their independently coded pre-treatment Adult Attachment Interview (AAI) with their narratives in a post-treatment interview about the relationship with

Correspondence: Jordan Bate, Ferkauf Graduate School of Psychology, Yeshiva University, 1165 Morris Park Ave. Bronx, NY 10461, USA.

E-mail: Jordan.bate@yu.edu

Citation: Talia, A., Miller-Bottome, M., Wyner, R., Lilliengren, P., $\&$ Bate, J. (2019). Patients' Adult Attachment Interview classification and their experience of the therapeutic relationship: are they associated? Research in Psychotherapy: Psychopathology, Process and Outcome, 22(2), 175-188. doi: 10.4081/ripppo.2019.361

Contributions: all authors contributed to designing the study and were involved in coding the data for this study. AT, MMB, JB wrote the manuscript and interpreted the results.

Acknowledgements: the authors want to express gratitude to the faculty at the Brief Psychotherapy Research Program, who along the therapist (the Patient Relationship Interview at Termination, PRI-T). The PRI-T was coded with the following three measures: i) The Patient Attachment to Therapist Rating Scale (PAT-RS), which assesses the quality of the patient's attachment relationship to the therapist; ii) the Coherence scale from the AAI, adapted for use on the PRI-T; and iii) the Patient Attachment Classification System (PACS), which measures generalized differences in how individuals convey their experiences and feelings. Results suggest that patients' AAI predicts how they experience, represent, and communicate about the therapeutic relationship at the end of treatment, as shown by the PAT-RS, the Coherence scale adapted for use on the PRI-T, and the PACS applied to the PRI-T. These findings lend support to Safran and others' hypothesis that patients' AAI-status plays a role in patients' representations of the relationship with the therapist.
\end{abstract} with Jeremy Safran provided the opportunities for this study, and the research assistants in Jeremy Safran's lab who transcribed the interviews for this project during the Fall 2012 in New York. The authors also want to thank Sam Bodian, who participated in this study as a PAT-RS rater, and Daniela Brambilla, who was an additional AAI coder.

Conflict of interest: the authors declare no potential conflict of interest.

Funding: the research reported on in this paper was supported by a NIMH grant, no. MH071768 and a grant from the American Psychoanalytic Association Fund for Psychoanalytic Research.

Dedication: this paper is dedicated to the memory of Jeremy D. Safran, who ideated this study and was a discussant in a symposium when it was first presented, at the 45th meeting of the Society for Psychotherapy Research (SPR) in Copenhagen, in 2014.

Received for publication: 15 December 2018.

Revision received: 18 March 2019.

Accepted for publication: 2 April 2019.

This work is licensed under a Creative Commons Attribution NonCommercial 4.0 License (CC BY-NC 4.0).

${ }^{\circ}$ Copyright: the Author(s), 2019

Licensee PAGEPress, Italy

Research in Psychotherapy:

Psychopathology, Process and Outcome 2019; 22:175-188

doi:10.4081/ripppo.2019.361
Key words: Relational therapy; Psychodynamic therapy; Attachment style; Adult Attachment Interview; Post-treatment interviews; Therapeutic relationship.

\section{Introduction}

From early on in his career, Jeremy Safran advanced the notion that the study of early development can deepen our knowledge of psychotherapy and the therapeutic relationship in particular. A well-known example of this view is the parallel he established between affective coordination in the mother-infant interaction and the rupture-repair process in psychotherapy (Safran, Muran, \& Samstag, 1994). Another example, perhaps less wellknown, was his more recent interest in integrating attachment research into the study of the therapeutic relationship (Bowman \& Safran, 2007). Starting around a decade ago, Safran led an initiative along with Chris Muran and Catherine Eubanks at the Brief Psychotherapy Research Program (BPRP) at Mount Sinai Beth Israel Medical Center whereby all patients were administered the Adult Attachment Interview (AAI, George, Kaplan, \& Main, 1996), the gold standard of adult attachment re- 
search, as well as a structured interview about the patienttherapist relationship at termination (the Patient Relationship Interview at Termination, PRI-T; Safran \& Muran, 2008). Safran believed that attachment theory offered a language for conceptualizing the qualities that can be transformative about therapeutic relationships, as well as a methodology for measuring the development of these qualities in psychotherapy.

Safran's interest in this topic was inspired by some central ideas in attachment theory and research. Following pioneering work by John Bowlby, attachment theorists maintain that all individuals develop generalized models of relationships based on their early experiences with caregivers, or attachment figures (Bowlby, 1969/1982). Individuals with adverse or inconsistent childhood experiences with their attachment figures are thought to develop negative expectations in intimate relationships and respond to emotional closeness with anxiety or withdrawal. Bowlby maintained that the therapist embodied some key features of an attachment figure, and as such could revive in the patient these expectations and patterns of responses (Bowlby, 1988). Building on this hypothesis, Safran believed that patients can experience the therapist over time as a different kind of attachment figure, one who is more responsive and available, which could create an opportunity to change patients' attachment-related schemata and representations.

Ample research evidence shows that individual differences in attachment predict several aspects of the therapeutic process, including how the patient communicates with the therapist in-session (Talia et al., 2014; Talia, Miller-Bottome, \& Daniel, 2017), how the therapist communicates with the patient (Talia, Muzi, Lingiardi, \& Taubner, 2018), and better treatment alliance and outcomes (see Slade, 2016). Despite the wealth of studies on this topic, however, it is not yet clear whether attachment differences also influence how patients subjectively experience the therapeutic relationship (Miller-Bottome, Talia, Safran, \& Muran, 2018). Previous studies in this area have almost always assessed patients' attachment through self-report measures (Mikulincer, Shaver, \& Berant, 2013). Such measures, while valuable in their efficiency, potentially over-emphasize attachment as a conscious process detectable at the level of the patient's perceptions. On the other hand, the one study to use an observer-based measure of attachment at intake to assess how attachment influences patients' experience of the therapeutic relationship only included a sample of ten patients, all of whom were diagnosed with borderline personality disorder (Diamond, Stovall-McClough, Clarkin \& Levy, 2003).

This paper reports the results of a study that investigated whether the pre-treatment AAI ratings of thirty outpatients with different diagnoses in Brief Relational Therapy (BRT, Safran \& Muran, 2000) are associated with their narratives in a post-treatment interview about the relationship with the therapist, the PRI-T. In particular, we analyzed patients' responses during the PRI-T to test whether patients' AAI ratings at intake predict: i) the attachment quality of the patient-therapist relationship as measured by the Patient Attachment to Therapist Rating Scale (PAT-RS), a validated rating instrument applied to post-treatment interviews (Lilliengren et al., 2014); ii) coherence of patients' narratives about the therapeutic relationship at the end of treatment, as measured by the Coherence scale, adapted for use on the PRI-T (Bate, Talia, \& Wyner, 2012) from the synonymous scale of the $\mathrm{AAI}$; and iii) how patients communicate about their experiences and feelings with the interviewer during the PRI-T, as measured by the Patient Attachment Coding System (PACS; Talia \& Miller-Bottome, 2015), an observer-based measure of attachment previously applied to analyze therapy sessions.

After a brief review of the evolution of ideas about individual differences in attachment representations, we summarize the background literature relevant to our three hypotheses. We then describe our sample and outline the methodology for our study. Finally, we present our findings and discuss their clinical and theoretical implications.

\section{Individual differences in attachment behavior and discourse}

The hallmark of contemporary attachment research is its emphasis on individual differences in how individuals engage in attachment relationships, which are relationships in which one seeks to maintain proximity to another preferred person (e.g., a parent, a spouse, a close friend, or one's therapist) when distressed or hurt. The study of such differences began with the work of Mary Ainsworth with the Strange Situation (SSP; Ainsworth, Blehar, Wall, $\&$ Waters, 1978). In the SSP, a laboratory procedure that consists of consecutive separations and reunions between caregiver and infant, infants usually seek proximity to the caregiver in one of three main ways. Some children, termed secure, actively seek closeness to the caregiver upon reunion and then readily resume exploring the room. Other children, termed avoidant, fail to seek proximity upon reunion and appear focused on exploring the room, while yet another group of children, termed ambivalent seems difficult to soothe and too distressed to explore independently. Bowlby hypothesized that these differences in infants' proximity seeking behaviors arise from differing expectations about the relationship as a source of protection, which he thought were encoded within mental structures called Internal Working Models (Bowlby, 1969/1982).

Inspired by these findings, Mary Main and colleagues developed the AAI in order to investigate whether attachment-related differences analogous to those observed in infants could be assessed in adults (Main, Kaplan, \& Cassidy, 1985). Main contacted a sample of parents whose children had been assessed in the Strange Situation and asked them to complete an hour-long, semi-structured in- 
terview concerning their early attachment experiences: the AAI. The central task of the interview was to recall and describe memories of attachment-related experiences in a coherent and collaborative manner (Hesse, 1996, 1999). Parents of secure infants provided narratives that were vivid, believable, and coherent as a whole. On the other hand, the narratives of parents of avoidant children were characterized by the seeming attempt to dismiss the impact of attachment-related experiences and were termed dismissing, while parents of ambivalent children appeared to display an ongoing preoccupation with their past attachment experiences and were termed preoccupied.

From these observations, Main, Goldwyn and Hesse (2002) developed the Adult Attachment Scoring and Classification System. According to this system, speakers are assigned a secure classification if their narratives in the AAI demonstrate openness and collaboration with the interview tasks and are clear and balanced as rated by the Coherence scale of the AAI. These speakers' narratives generally reflect a valuing of attachment-related experiences, an ability to access attachment-related cognitions, and a general ease in discussing and reflecting on these topics. By contrast, speakers are assigned a dismissing classification if their narratives are characterized by the absence of specific memories from their childhood or unsubstantiated idealized representations of their attachment relationships. Finally, speakers are assigned a preoccupied classification if their narratives convey a sense that they are still struggling with attachment-related memories, as demonstrated by either disproportionate anger for their parents' past offenses, or by a confusing discourse style, full of vagueness and digressions. In the years since, the AAI has become the gold standard assessment of adult attachment research (Bakermans-Kranenburg \& van IJzendoorn, 2009), and its validity has been demonstrated by a recent meta-analysis involving more than 78 different studies (Verhage et al., 2016).

\section{The patient-therapist relationship as an attachment relationship}

Drawing from Bowlby's intuition that the therapeutic relationship may be considered an attachment relationship in many respects, as well as from empirical work in its support (Parish \& Eagle, 2003), many clinicians and psychotherapy researchers have made the additional assumption that the infant attachment patterns in the SSP have an obvious analogue in the therapy room (Mallinckrodt, 2010). Departing from Main's work on discourse and coherence, these authors view attachment styles in therapy as reflecting differences in comfort with closeness and differentiation in the attachment-like relationship with the therapist (Marmarosh, 2015). In this perspective, secure patients are likely to experience their therapists as capable of providing care and comfort; avoidant patients will fear intimacy and experience discomfort with getting closer to the therapist; and preoccupied patients will fear rejection and be preoccupied with abandonment (Wallin, 2007).

Following these views, Mallinckrodt and his colleagues developed the Client Attachment to Therapist Scale (CATS; Mallinckrodt, Gantt, \& Coble, 1995), a 36item questionnaire with three subscales that capture secure, avoidant-fearful, and preoccupied-merger components of attachment to the therapist. A recent metaanalysis shows that the CATS subscales have good internal consistency and test-retest reliability, and they correlate in expected ways with measures of adult attachment, session depth and smoothness, and therapeutic alliance (Mallinckrodt \& Jeong, 2015).

Despite these important findings, using patient-reported questionnaires to assess patients' attachment to the therapists might have some limitations. First, since the CATS rates the quality of the patient-therapist attachment relationship, it is difficult to distinguish a lack of attachment that may be typical of the early phases of treatment from an attachment to the therapist that is insecure (Allen, Stein, Fonagy, Fultz, \& Target, 2005). Second, because attachment representations are unconscious, a patient completing a questionnaire may not competently report on his or her own attachment dispositions towards the therapist. This concern seems to be supported by metaanalytic evidence that shows that self-report and observerreport measures of attachment have only small to trivial associations with each other (Roisman et al., 2007).

For these and other reasons, Lilliengren and colleagues have developed and validated an interview-based rating system, the PAT-RS (Lilliengren et al., 2014), which yields a rating of the quality of patient-therapist attachment based on how patients describe their experience of the therapeutic relationship. Because its approach is based on independent observer-ratings of patients' narratives, the PAT-RS was designed to overcome some of the limitations of self-report questionnaires of attachment. The PAT-RS was developed for any type of interview in which patients describe their experience of their relationship with the therapist and assesses the patient-therapist attachment relationship along four dimensions: Security (which reflects low anxiety and low avoidance, as well as a balance between autonomy and dependence in the therapeutic relationship), Hyperactivation (which reflects being preoccupied with worry about abandonment and lack of understanding and care from the therapist), Deactivation (which reflects the patient keeping an emotional distance in the therapeutic relationship), and Disorganization (which reflects a simultaneous presence of high anxiety and high avoidance).

One study has supported the usefulness of the PAT-RS (Lilliengren, Falkenström, Sandell, Mothander, \& Werbart, 2015), finding that secure attachment to the therapist at termination as measured by this instrument was associated with improvement in symptoms, global functioning, and interpersonal problems; the relationships between the PAT-RS and symptoms and global functioning were 
maintained even after controlling for the level of the therapeutic alliance. In contrast with the approach of the AAI, which emphasizes generalized tendencies in representing relationships, the PAT-RS is a relationship-specific measure that focuses on the quality of the therapeutic relationship. However, because we assumed that patients' overall AAI classification will have an influence on their experience of the therapeutic relationship as assessed by the PAT-RS, in this paper we test the association between these two measures.

\section{States of mind with respect to the attachment to the therapist: the role of coherence}

A different hypothesis about attachment-related differences in the patient-therapist relationship originates from the work of Mary Main and her colleagues with the AAI (Main et al., 1985). Although initially Main and colleagues emphasized the link between narrative and parents' state of mind with respect to early attachment experiences, in the years since researchers have devised several other interviews, designed to assess narrative coherence with regards to other attachment relationships within different populations (e.g., Current Relationship Interview, CRI, Crowell, Treboux, \& Waters, 2002; Parent Development Interview, PDI, Slade, 2005; Working Model of the Child Interview, WMCI, Zeanah, Benoit, Barton, \& Hirshberg, 1996). Other researchers have measured narrative coherence in psychotherapy, investigating whether differences in coherence emerge similarly in psychotherapy sessions as they do in the AAI, and if coherence increases during therapy (Ammaniti, Dazzi, \& Muscetta, 2008; Daniel, Poulsen, \& Lunn, 2016; Holmes, 2001; Levy, et al., 2006; Muscetta, Dazzi, De Coro, Ortu, \& Speranza, 1999; Samstag et al., 2008; Stovall-McClough \& Cloitre, 2003; Tmej, Fischer-Kern, Doering, Alexopoulos, \& Bucheim, 2018).

In a similar vein, Diamond and her colleagues developed and implemented an interview based on the AAI in order to assess patients' representations of their therapists: the Patient-Therapist Adult Attachment Interview (Diamond et al., 2003). The first sixteen questions of the PTAAI, as well as the rating and classification system that Diamond and colleagues developed for this interview, are based directly on Main's and colleagues' work on the AAI. If the therapist can be considered an attachment figure, they hypothesized, patients of different attachment classifications may recall and reflect upon the therapeutic relationship in ways that are influenced by their state of minds with respect to attachment. In particular, Diamond and her colleagues posited that narrative coherence in the PT-AAI might be a function of patients' flexible access to a range of different thoughts and feelings about the therapist, without interference of defense mechanisms. They also argued that incoherence in this interview may reflect the activation of defenses (such as repression and rationalization), or the emergence of multiple models of the therapeutic relationship (marked for example by idealization), both of which may occur when the patient is confronted with conflictual feelings about the therapist.

In their study of ten patients with Borderline Personality Disorder (BPD), Diamond and her colleagues administered the AAI at the 4-months mark and at termination of a yearlong course of Transference Focused Psychotherapy (Kernberg, Yeomans, Clarkin, \& Levy, 2008) and the PT-AAI at termination only. The main finding was the partial concordance between patients' AAI classifications and their PTAAI classifications at termination. These results indicate that, at least in this sample, patients displayed similar narrative tendencies when describing their relationship with their caregivers as they did when describing their experiences with their therapist. Diamond and colleagues concluded that the PT-AAI in combination with the AAI given over the course of therapy may be useful in tracking aspects of the transference as it unfolds over time, particularly in identifying the specific attachment states of mind with respect to parents that are recapitulated with the therapist. (Diamond et al., 2003, p. 240).

The current study builds on the results obtained by Diamond and examines the relationship between patients' AAI ratings at intake and their coherence (rated according to an adaptation of the Coherence scale of the AAI to the PRI-T) in a post-treatment interview similar to the PTAAI, the PRI-T. Rather than comparing categorical classifications on the two interviews as Diamond and colleagues did, in order to enhance reliability and statistical power we limited ourselves to evaluating the dimensional scores of the AAI and the Coherence scale adapted for use on the PRI-T.

\section{The Patient Attachment Coding System: attachment-related differences in verbal communication}

Recent work in psychotherapy research has used the fine-grained analysis of language and communication that characterizes the AAI to explore whether patients' attachment differences impact their in-session behavior with the therapist (Talia et al., 2014; Talia, Miller-Bottome, \& Daniel, 2017). Influenced by the work of ordinary language philosophy, the authors of the PACS proposed to analyze language as a social behavior that inevitably affects relationships. They hypothesized that patients with different attachment classifications reveal distinct patterns in their use of language to regulate closeness and connection with their listener. They then conducted a qualitative analysis to examine the characteristics of these patterns.

In this qualitative analysis, it was discovered that attachment classifications manifest in patients in psychotherapy as distinct ways of communicating about their experience and internal states. Secure patients convey their present experience openly and allow the therapist to participate in defining and elaborating it. For example, secure patients disclose their emotions in the here and now and share vivid narratives of past experiences that clearly 
convey their feelings in the present. Secure patients also communicate their feelings and needs in the therapeutic relationship, and they share their present intentions, autonomous reflections, and positive experiences. These speech acts, or markers, are rated on the PACS Proximity seeking, Contact Maintaining, and Exploring scales. Avoidant patients, on the other hand, tend to decline requests to express their feelings, or are reluctant to describe their experiences in sufficient detail. These markers in the PACS are scored on the Avoidance scale. Finally, preoccupied patients tend to disregard the therapist's interventions and share their experience in a one-sided, exaggerated, or confusing way that leaves little room for the therapist to respond. These patterns of communication, rated on the Resistance scale of the PACS, tend to limit the extent to which the therapist is able to make meaning of the patients' experience.

Studies using the PACS on different samples and in a range of therapeutic modalities have confirmed that AAI classifications predict marked differences in patients' insession communication, and that by analyzing such differences in a single session one can predict patients' AAI classifications with precision (Talia et al., 2017). One scale of the PACS, the Exploring scale, has also shown to be closely associated with patients' independently obtained rating of mentalizing in the context of the AAI (Talia et al., 2018). Finally, accumulating evidence shows that PACS security predicts greater resolution of alliance ruptures (Miller-Bottome, Talia, Eubanks, Safran, \& Muran, 2019), as well as greater physiological synchronization between patient and therapist (Kleinbub, Talia, \& Palmieri, submitted for publication in 2019).

Research using the PACS is only in its infancy, and the observations that have emerged in these studies raise many new questions. In particular, empirical studies are needed in order to establish whether the differences in communication outlined by the PACS are specific to psychotherapy, or whether they emerge in other relationships as well. A related question is whether these differences might be reliably assessed outside of psychotherapy (Talia et al., 2017), for example in diagnostic or post-treatment interviews. If this is the case, patients' patterns of communication as assessed by the PACS may influence how patients' recall and reflect on the therapeutic relationship, beyond their observed in-session behavior and discourse.

The present study presents a preliminary effort to answer this question. In particular, in the current study we use the PACS to measure how the patient communicates his or her experiences and feelings while discussing a set topic with an interviewer (i.e., the therapeutic relationship). As argued elsewhere (Miller-Bottome et al., 2018), we expect such capacity to be a trait or characteristic that is relatively independent from the specific content or quality of the experience under discussion. In other words, we assume a secure patient may be able to communicate with their listener with their characteristic openness and recep- tivity, regardless of the content of the experience they are describing, and whether or not they are feeling understood by the listener. Because the way in which speakers communicate impacts what content can be conveyed, however, we also expect the PACS scales to be associated with the other measures included in this study.

\section{Aims and hypotheses}

The aim of this study was to investigate the relationship between patients' pre-treatment AAI ratings and their experience of the therapeutic relationship as recounted during the PRI-T and assessed on the transcripts of this interview via three different ratings systems: the PAT-RS, the Coherence scale adapted for use on the PRI-T, and the PACS. Because of the relatively small sample size of this study, we mainly relied on dimensional continuous scores of attachment rather than categorical classifications. As we shall describe in greater detail in the Methods section, we used the AAI Coherence scale as a measure of patients' AAI security and computed dimensional scores of dismissiveness and preoccupation (from here on, AAI Dismissing score and AAI Preoccupied score, respectively) following Haydon and colleagues' method (Haydon, Roisman, Marks, \& Fraley, 2011). However, because the PACS (in contrast with the PAT-RS and the Coherence scale adapted for use on the PRI-T) yields main attachment classifications in addition to dimensional scales, we also compared the PACS classifications with patients' pretreatment AAI main classifications.

Our specific hypotheses were as follows. First, we tested if patients' AAI ratings predict the quality of the patient's attachment relationship with the therapist as assessed with the PAT-RS applied to the PRI-T. In particular, we hypothesize that the Coherence scale of the AAI will be related to the Secure factor of the PAT-RS, while the AAI Dismissing score and the AAI Preoccupied score will be related to the PAT-RS Deactivating and Hyperactivating factors, respectively. Since the PAT-RS is a relationship specific measure and is dependent on patients' pre-treatment attachment representations as well as the specific interaction with the therapist, we hypothesize the associations between patients' pre-treatment AAI and post-treatment PAT-RS will only be moderate.

Second, we tested if the AAI Coherence scale predicted the capacity of the patient to be coherent and provide examples as requested to support the description of the therapeutic relationship, as rated by the Coherence scale adapted for use on the PRI-T. In particular, we hypothesized that the Coherence scale of the AAI will have a significant strong association with coherence in the PRIT, while both the AAI Dismissing score and the AAI Preoccupied score will be inversely related to it.

Finally, we tested if patients' pre-treatment AAI predicts their communication with the interviewer during the PRI$\mathrm{T}$ as assessed by the PACS. In particular, we hypothesize that patients' AAI Coherence scale will be strongly associ- 
ated with patients' PACS global security during the PRI-T, while the AAI Dismissing score and the AAI Preoccupied score will be related to PACS Avoidance and Resistance, respectively. Because both the AAI and the PACS coding systems yield a categorical classification of patients' discourse, we also hypothesized that their respective classifications of the same patients will be highly associated.

\section{Methods}

\section{Participants}

This study was conducted at the BPRP at the Mount Sinai-Beth Israel Medical Center in New York City. At the time these data were collected, patients were recruited through local and online advertising, referrals made by hospital staff, and contact with other providers within the community, such as university counseling centers. Participants were included in this Research Program if they are 18-65 years old, proficient in English, and willing to have therapy sessions recorded for research purposes and to complete other assessment parameters. All patients were first screened in a brief phone interview and then assessed with the Structured Clinical Interview for DSM-IV Axis I and II disorders. Patients were excluded if there was evidence of a substance use disorder, suicidality, psychosis, history of any problem with the law, or history bipolar I or II disorder. Patients stabilized on psychiatric medication for at least three months prior to intake (i.e., no report of dosage adjustment during that time) were included, while those whose psychiatric medications had been adjusted within the last three months were excluded from the study and referred elsewhere for treatment.

For this study, all patients with a fully recorded AAI and PRI-T who completed 30 sessions of BRT by the end of 2015 were included. The sample was comprised of 30 adults (17 female, 13 male), age 21-68 years (mean=39.72, $\mathrm{SD}=13.06$ ). Additional demographic information is summarized in Table 1 (the data from one participant is missing). Clinicians evaluated patients' overall functioning at baseline using the Global Assessment Scale (GAS). The scale ranges from 0 to 100 , with each $10-$ point span describing a different level of functioning, and higher scores indicating better functioning overall. The mean GAS rating at the beginning of treatment was 62.5 $(\mathrm{SD}=10.3)$, with ratings seemingly fitting a normal distribution, with skewness of $0.46(\mathrm{SE}=.47)$ and kurtosis of $0.43(\mathrm{SE}=.92)$.

\section{Measures}

\section{The Adult Attachment Interview}

The AAI (George et al., 1996) consists of 20 questions focusing on the participants' early experiences with their parents or other primary caregivers. One of its core components is a question that asks people to list five words that describe their early relationship with each of their parents and then elicits specific memories or incidents associated with those words. Participants are also asked whether and why they felt closer to one parent or the other; whether they ever felt rejected during childhood; whether their parents threatened them in any way during childhood; why they think their parents may have acted as they did at the time; how these experiences may have impacted their adult personalities; and whether they have experienced any major losses, during or since childhood.

All AAIs were coded with the Adult Attachment Scoring and Classification System (Main, Goldwyn, \& Hesse, 2002). This system yields five possible main classifications: secure/autonomous, dismissing, preoccupied, unresolved/disorganized, and cannot classify, which are assigned on the basis of the ratings on a number of continuous scales. High ratings on AAI Coherence of Transcript scale (hereafter referred as simply AAI Coherence) lead to assigning a Secure classification. High ratings on the AAI scales Idealization of Parent (which rates the extent to which speakers offer a glib, positive image of their parents that they fail to support with specific memories)

Table 1. Frequencies for demographic characteristics.

\begin{tabular}{lc}
\hline Demographic characteristic & N (\%) \\
\hline Marital Status & \\
Single/Never Married & $18(60.0)$ \\
Married & $6(20.0)$ \\
Divorced/Separated & $5(16.7)$ \\
\hline Education & \\
Some College & $6(20.0)$ \\
College Graduate & $14(43.3)$ \\
Some Post-Graduate & $1(3.3)$ \\
Graduate Degree & $9(30.0)$ \\
\hline Employment Status & \\
Employed & $23(76.7)$ \\
Unemployed & $6(20.0)$ \\
\hline Race/Ethnicity & \\
White (non-Hispanic) & $22(73.3)$ \\
Hispanic & $1(3.3)$ \\
Black & $1(3.3)$ \\
Other & $5(16.7)$ \\
\hline Axis I Diagnosis & \\
Major Depression, Recurrent & $7(23.3)$ \\
Major Depression, Single Episode & $4(13.3)$ \\
Dysthymia & $3(10.0)$ \\
Past Major Depressive Episode & $2(6.7)$ \\
Generalized Anxiety Disorder & $2(6.7)$ \\
Social Phobia & $1(3.3)$ \\
Depressive Disorder NOS & $1(3.3)$ \\
Anxiety Disorder NOS & $1(3.3)$ \\
Other Diagnosis (include V codes) & $4(13.3)$ \\
Deferred Diagnosis & $4(13.3)$ \\
\hline Axis II Diagnosis & \\
Personality Disorder & $9(30.0)$ \\
No Diagnosis & $18(60.0)$ \\
Missing Data & $3(10.0)$ \\
\hline & \\
\hline & \\
\hline
\end{tabular}


and Insistence on Lack of Recall (which rates the speaker's claims of being unable to remember their childhood experiences), lead to assigning a Dismissing classification. High ratings on the AAI scales Involving or Preoccupying Anger (which rates the extent to which the speaker expresses anger in ways that indicate ongoing preoccupation with past attachment experiences) and Passivity or Vagueness of Discourse (which rates the extent to which the speaker uses overly vague or confusing language) lead to assigning a Preoccupied classification. For the purposes of this study, we did not include the Unresolved classification or the Cannot Classify classification because we did not have hypotheses specifically related to them, and in the analyses based on categorical classifications we used a forced three-way classification.

Beyond the final classifications of the AAI, its underlying dimensions have been conceptualized either as a combination of a secure-insecure dimension and a deactivation-hyperactivation dimension (Kobak, Cole, FerenzGillies, \& Fleming, 1993; Waters, Treboux, Fyffe, \& Crowell, 2005) or as a combination of a dismissing dimension and a preoccupied dimension (e.g., Haydon et al., 2011; Haydon, Roisman, \& Burt, 2012). In this study, in addition to using the AAI categorical classifications described above, we refer to Haydon's et al.'s two-dimensional conceptualization, which has been replicated by other studies (e.g., Dykas, Woodhouse, Jones, \& Cassidy, 2014; Haltigan et al., 2014; Martin, Raby, Labella, \& Roisman, 2017; Tarabulsy et al., 2012). We calculated Dismissing and Preoccupied dimensional scores for each patient by first multiplying the standardized scores of each of the relevant AAI rating scales (e.g. Idealization of Parent, Involving Anger, etc.) by Haydon et al.'s coefficient weights, and then averaging the scores so obtained for each dimension to yield an AAI Dismissing score and an AAI Preoccupied score. We then analyzed the AAI data in terms of raw scores on these two dimensions, as well as on the AAI Coherence scale, which we used as a measure of overall security.

The three-way distribution of the AAI classifications in this outpatient sample did not differ significantly from the norm distribution of attachment representations in non-clinical samples reported by Bakermans-Kranenburg and van IJzendoorn (2009), $\chi 2(2, \mathrm{~N}=30)=1,810, \mathrm{P}=.405$. Similarly, the distribution of the combined unresolved/cannot classify group $(\mathrm{N}=6)$ vs the combined not-unresolved group did not differ significantly from its norm distribution: $\chi^{2}(1, \mathrm{~N}=30)=.081, \mathrm{P}=.776$.

\section{The Patient Relationship Interview at Termination}

The PRI-T (Safran \& Muran, 2008), modeled on George, Kaplan, and Main's (1996) AAI and Diamond et al.'s (2003) PT-AAI, consists of 21 questions that focus on patients' experiences with their therapists during the course of treatment. Like the PT-AAI, the first 16 questions are identical to those of the AAI, except for minor adjustments in the terms used. Questions about how the patient felt about the therapist and how the patient thinks the patient feels about them are also included. When compared with the PT-AAI, the PRI-T also includes questions that probe for experiences within the patient-therapist relationship related to Safran and Muran's (2000) conceptualization of the therapeutic alliance, including asking patients about ruptures and the extent to which they were repaired. For example, the PRI-T asks: Did you experience any tension, problems, conflicts, or misunderstandings in your relationship with your therapist? and then prompts the patient to recount specific examples and further explore those experiences. The PRI-T also prompts patients to discuss what they expected or fantasized about treatment, what they found most helpful, and what aspects of treatment were not so helpful.

Similar to the AAI, the PRI-T aims to surprise the unconscious of interviewees, calling upon them, repeatedly, to explain, contradict, support, or leave unsubstantiated their claims. However, unlike Diamond's PT-AAI, an attachment classification system was not developed for the PRI-T. Instead, research at the BPRP has used the PRI-T primarily to look at patients' reflective functioning (Katzow, 2011). This is the first published study in which the PRI-T was coded to evaluate attachment using three different rating systems, which we shall describe below.

\section{The Patient Attachment to the Therapist-Rating Scale}

The purpose of the PAT-RS (Lilliengren et al., 2014) is to assess the quality of the patient's attachment to his or her therapist based on patients' narratives of the therapist as a person, how the patient experienced the therapeutic relationship, and how he or she reacts to attachment-related issues in the therapeutic relationship (i.e., closeness, separation, etc.). Following the two-dimensional model of attachment (Mikulincer et al., 2013), the PAT-RS was designed to assess the quadrants of attachment relatedness in four subscales: Security (low anxiety, low avoidance), Hyperactivation (high anxiety, low avoidance), Deactivation (low anxiety, high avoidance), and Disorganization (high anxiety, high avoidance).

The PAT-RS was designed for use on any interview material in which patients describe their experience of their relationship with the therapist and preliminary data suggests acceptable reliability and validity using a post-therapy interview similar to the PRI-T (Lilliengren et al., 2014, 2015). When rating an interview with the PAT-RS, the rater evaluates how much the patient experienced the therapeutic relationship as secure, deactivating (corresponding to dismissing), hyperactivating (corresponding to preoccupied), and disorganized (corresponding to a mix of the latter two) in relation to nine components (e.g., secure base, stronger and wiser, safe haven, proximity seeking, particularity, responsiveness, strong feelings, separation anxiety and mental representation). The rater must rate on a 5-point Likert scale the degree to which a patient describes each of these 
components in a way that is similar to four prototypes (secure, deactivating, hyperactivating, and a disorganized prototype). Next, the rater sums the ratings of each prototype throughout the nine components of the instrument and divides it by nine (i.e., the total number of components rated for that interview), in this way obtaining a final score on four subscales (secure, deactivating, hyperactivating, and disorganized) ranging from 1 to 5 .

\section{The Coherence Scale adapted for use on the Patient Relationship Interview at Termination}

The Coherence Scale adapted for use on the Patient Relationship Interview at Termination (Bate et al., 2012) aims to measure the overall narrative coherence in the patient's representation of the therapist - i.e., whether the PRI-T transcript presents a well-organized and logical flow of ideas and feelings about the therapeutic relationship or the therapist. Similar to its equivalent in the AAI, the Coherence scale adapted for use on the PRI-T is based on Grice's cooperative maxims (Main et al., 2002). The scale takes into account both positive indices of coherence, or markers of consistency and collaboration, and violations of Grice's maxims of rational discourse (quality, quantity, relation, and manner). Collaboration, openness, and the ability to construct a clear, credible story that the interviewer can follow are seen as positive indicators of coherence that can elevate one's score on this measure. Violations of quantity (i.e. providing too much or too little information), quality (i.e. failing to provide evidence of what is said), relevance (i.e. addressing topics unrelated to the interview questions), and manner (i.e. speaking ambiguously, vaguely, or obscurely) are markers of incoherence.

The main difference between the AAI Coherence scale and the Coherence scale adapted for use on the PRI$\mathrm{T}$ is that in the latter raters assign a rating from 1 to 9 to each maxim individually, with higher scores indicating higher levels of coherence, based on the number and severity of violations. These subscales are then used to determine the final score of the Coherence scale adapted for use on the PRI-T, which is given a rating on a Likert scale from 1 to 9 . Highly coherent narratives are not only consistent and collaborative but have a quality of freshness and comfort with the topic regardless of whether the actual memories and experiences themselves were positive or negative. Narratives rated low in coherence may present confusing or contradictory pictures of the therapeutic relationship and a lack of openness and collaboration. The scoring system provides detailed guidelines and specific examples to aid in the determination of a specific rating.

The primary reason for developing this method of coding was to make it easier to train coders to reliability. While the authors of the coding system (Bate, Wyner, Talia) were all trained by Mary Main and Erik Hesse and obtained reliability in coding of the AAI, the Coherence coding system adapted for use on the PRI-T was designed to train coders who had not previously received AAI training. Therefore, the use of subscales with specific markers provided clear guidance to train and provide feedback to new coders.

\section{The Patient Attachment Coding System}

The PACS (Talia et al., 2017) has been originally validated to assess patients' in-session attachment based on any single psychotherapy session transcribed verbatim, regardless of the topics discussed or the therapeutic modality. When applying the PACS, the transcript is rated as a whole without segmenting the text, and the coder identifies the occurrence of any number of 50 discursive markers described in a coding manual (Talia \& MillerBottome, 2015) as they occur in patients' speech turns. Each marker serves a distinct function in communication and can be assigned to a word, to a single utterance or to a whole speech turn. The coder gives a rating from 1 to 7 in .5 increments on eleven subscales, based on both the frequency and intensity of the markers belonging to each subscale. The ratings of the subscales lead to assign a rating on five main scales.

In this study, we used four out of the five PACS main scales: Proximity seeking, which comprises markers where the speaker discloses his or her present distressful experience (e.g., the patient criticizes the unloving treatment received by someone, or describes a vivid episode in which he or she was hurt); Exploring, which comprises markers where the speaker discloses positive feelings or experiences and autonomous reflections; the Avoidance scale, which is associated with an evasion of inquiries into the speaker's positive and negative experience, and with a minimization or deferment of any mental state previously conveyed (e.g., the speaker affirms that he or she has no right to complain; chuckles about his or her own distress, etc.); and the Resistance scale, which captures discourse markers that enlist the listener's agreement with the speaker's views or otherwise restrict the listener's capacity to disagree, for example by being vague or excessively detailed. We did not use the PACS Contact maintaining scale, which rates instances in which the speaker discusses how their listener has emotionally affected them (e.g., when a patient expresses gratitude to the therapist for his or her support during a session, or notes the emotional resonance of a particular therapist remark or intervention), because we did not expect such markers to occur frequently with an interviewer in a semistructured interview.

Finally, a global score on the PACS Security scale is obtained by a simple algorithm that is based on the rating of the PACS main scales. A three-way classification of patient's attachment is obtained by observing the overall configuration of the scales. A rater classifies the patient's in-session attachment as secure if Proximity Seeking or Exploring are higher than the other scales, while an avoidant or preoccupied classification is assigned if Avoidance or Resistance are higher, respectively. 


\section{Procedure}

Each patient in this study received 30 sessions of BRT (Safran \& Muran, 2000), administered on a weekly basis at the Brief Program. BRT is a short-term, relational treatment that focuses on the here-and-now of the patient-therapist relationship and on attending to subtle shifts or ruptures in the therapeutic alliance that occur moment to moment. Treatments were conducted by doctoral level psychology externs or interns, or by psychiatry interns or residents, under weekly group supervision by licensed clinical psychologists.

Before treatment, trained research assistants administered the Structured Clinical Interview for the Diagnostic and Statistical Manual-IV (SCID-IV) and the AAI to each participant. After treatment, trained research assistants administered the PRI-T to each patient, along with several other standard outcome measures that were not part of this study. All interviews were transcribed verbatim following similar guidelines to those indicated by Main for the AAI (Main et al., 2002). All AAIs were coded by one of four reliable coders. Inter-rater agreement on three-way AAI classifications was calculated on $23.3 \%$ of the AAIs, and it was .88.

Seven trained coders (one doctoral and six master students) who were blind to subject identity and AAI classification rated the PRI-T transcripts using the PAT-RS (three coders), the Coherence scale adapted for use on the PRI-T (two coders), and the PACS (two coders). In order to test inter-rater reliability of the scales, $60 \%$ of the transcribed interviews were coded by all three PAT-RS coders, and $40 \%$ were coded by two coders each with the Coherence scale adapted for use on the PRI-T and the PACS. Two-way random single measures intra-class correlation coefficients were excellent for the Security and Deactivation scales of the PAT-RS (.80 and .86), and moderate for the Hyperactivation scale (.56). ICC was poor for the Disorganization scale $(.27)$, which was thus excluded from the following analyses. Two-way random average measures intra-class correlation coefficients were excellent for the Coherence scale adapted for use on the PRI-T (.85), and for all four main scales of the PACS $(.80, .84, .86$, $.88)$. The agreement between two raters at the level of the PACS classifications was $100 \%(\kappa=1, \mathrm{P}<.001)$.

Minimums, maximums, means and standard deviations for ratings on the AAI as well as all three coding systems applied to the PRI-T are reported in Table 2.

\section{Results}

Zero-order correlations between all the instruments used in our study were computed and are reported in Table 3. As hypothesized, patients' AAI Coherence scale had a significant albeit small association with the Secure scale of the PAT-RS, and patients' AAI Dismissing score had a moderate association with the Deactivating scale on the PAT-RS. Contrary to our hypotheses, patients' Preoccupied score on the AAI was not significantly associated with the Hyperactivating scale of the PAT-RS.

As hypothesized, patients' Coherence ratings on the AAI had also a significant association with patients' Coherence ratings on the PRI-T, and the AAI Dismissing score had a significant, inverse relationship with patients' Coherence ratings on the PRI-T. Contrary to our hypotheses, patients' Preoccupied score on the AAI was not significantly associated with patients' Coherence ratings on the PRI-T.

Finally, as per our hypotheses, patients' Coherence ratings on the AAI had a significant strong association with patients' PACS Security ratings on the PRI-T, the AAI Dismissing score had a significant strong association with

Table 2. Descriptives of the variables included in this study.

\begin{tabular}{|c|c|c|c|c|c|c|c|c|}
\hline & \multicolumn{2}{|c|}{ Secure $(n=15)$} & \multicolumn{2}{|c|}{ Dismissing $(\mathbf{n}=10)$} & \multicolumn{2}{|c|}{ Preoccupied $(n=5)$} & \multicolumn{2}{|c|}{ Total $(\mathbf{N}=30)$} \\
\hline & Min-Max & Mean (SD) & Min-Max & Mean (SD) & Min-Max & Mean (SD) & Min-Max & Mean (SD) \\
\hline \multicolumn{9}{|l|}{ AAI dimensional scores } \\
\hline AAI Coherence Scale & $5.5-8.5$ & $7.0(1.0)$ & $1.0-4.0$ & $2.8(0.8)$ & $2.0-3.0$ & $2.6(0.5)$ & $1.0-8.5$ & $4.9(2.3)$ \\
\hline Dismissing factor & $-0.7-0.2$ & $-0.4(0.3)$ & $-0.5-1.3$ & $0.7(0.5)$ & $-0.5--0.1$ & $-0.3(0.2)$ & $-0.7--0.3$ & $0.0(0.6)$ \\
\hline Preoccupied factor & $-0.4-0.7$ & $-.09(0.3)$ & $-0.4-0.2$ & $-0.2(0.2)$ & $0.8-1.3$ & $1.0(0.2)$ & $-0.4-1.3$ & $0.1(0.5)$ \\
\hline \multicolumn{9}{|l|}{ Rating scales on PRI-T } \\
\hline PAT-RS Security & $1.8-4.4$ & $3.4(0.9)$ & $1.2-4.3$ & $2.3(1.0)$ & $1.7-4.2$ & $3.2(1.1)$ & $1.2-4.4$ & $3.0(1.1)$ \\
\hline PAT-RS Deactivation & $1.0-2.6$ & $1.6(0.5)$ & $1.3-4.4$ & $3.4(1.2)$ & $1.1-2.3$ & $1.8(0.5)$ & $1.0-4.4$ & $2.2(1.1)$ \\
\hline PAT-RS Hyperactivation & $1.0-4.1$ & $1.9(1.0)$ & $1.0-3.0$ & $1.4(0.6)$ & $1.0-3.2$ & $1.8(0.9)$ & $1.0-4.1$ & $1.7(0.8)$ \\
\hline PAT-RS Disorganization & $1.0-1.8$ & $1.1(0.2)$ & $1.0-3.6$ & $1.4(0.9)$ & $1.0-1.3$ & $1.1(0.1)$ & $1.0-3.6$ & $1.2(0.5)$ \\
\hline PRI-T Coherence Scale & $5.0-8.0$ & $6.6(0.8)$ & $3.0-6.0$ & $4.3(0.9)$ & $4.0-6.0$ & $5.0(0.7)$ & $3.0-8.0$ & $5.6(1.4)$ \\
\hline PACS Security (Global) & $4.5-6.5$ & $5.3(0.7)$ & $1.5-5.5$ & $2.8(1.3)$ & $1.5-2.5$ & $2.1(0.5)$ & $1.5-6.5$ & $3.9(1.7)$ \\
\hline PACS Proximity Seeking & $1.0-6.0$ & $2.7(1.4)$ & $1.0-3.0$ & $1.7(0.8)$ & $1.0-3.5$ & $2.0(1.0)$ & $1.0-6.0$ & $2.3(1.2)$ \\
\hline PACS Exploring & $4.0-6.0$ & $5.1(0.7)$ & $1.0-5.5$ & $2.6(1.4)$ & $1.0-2.5$ & $1.6(0.7)$ & $1.0-6.0$ & $3.6(1.8)$ \\
\hline PACS Avoidance & $1.0-3.0$ & $1.6(0.7)$ & $1.0-6.0$ & $4.5(1.7)$ & $1.0-3.0$ & $1.7(0.8)$ & $1.0-6.0$ & $2.5(1.7)$ \\
\hline PACS Resistance & $1.0-4.5$ & $2.5(1.3)$ & $1.0-4.5$ & $1.6(1.1)$ & $4.5-6.5$ & $5.6(0.8)$ & $1.0-7.0$ & $2.9(1.9)$ \\
\hline
\end{tabular}

SD, standard deviation; AAI, Adult Attachment Interview; PRI-T, Patient Relationship Interview at Termination; PAT-RS, Patient Attachment to Therapist Rating Scale; PACS, Patient Attachment Coding System. 
PACS Avoidance ratings on the PRI-T, and the AAI Preoccupied score had a significant strong association with PACS Resistance ratings on the PRI-T. The three-way agreement between patients' pre-treatment AAI classification and the PACS coding of a post-treatment interview was excellent $(\kappa=.84$, Table 4$)$.

\section{Discussion and Conclusions}

In the final decade of his career, Jeremy Safran became interested in applying attachment theory to better understand the developmental roots of the therapeutic relationship and the role of secure attachment in the change process. The current study was an attempt to tie together the threads of this vision and to add to the growing tapestry of research on attachment and the therapeutic relationship. The results of this study support the hypothesis that patients' attachment representations of their caregivers influence how patients experience, represent, and communicate about the therapeutic relationship at the end of treatment, as shown by the significant relationship between patients' pre-treatment AAI on one hand and the PAT-RS, the Coherence scale adapted for use on the PRIT, and the PACS applied to the PRI-T on the other. Although the three coding systems included in this study attend to different aspects of patients' discourse, their associations with each other suggest that patients' attachment to the therapist, their coherence in discussing the therapeutic relationship, and their openness and autonomy in communication with an interviewer are related in meaningful ways. Finally, our study has some noteworthy methodological implications, namely that the PACS, the Coherence scale adapted for use on the PRI-T, and the PAT-RS are reliable and may be used to assess patients' attachment on an interview about the therapist at the end of treatment, without administering the AAI.

All of our hypotheses were supported with the exception that the AAI Preoccupied score was found to be significantly associated only with the PACS scales, and not with the PAT-RS or the Coherence scale adapted for use on the PRI-T. The lack of significant associations between the AAI Preoccupied score and the PAT-RS suggests that the view of preoccupied patients as hyper-activating in their relationship with their therapist - which is at the basis of the attachment style literature that informed the construction of the PAT-RS - may not be entirely correct. Perhaps patients with preoccupied states of mind on the AAI sometimes form secure attachment relationships with the therapist; or their relationship with their therapist may be characterized by qualities other than hyper-activation, for

Table 3. Cross-correlations between the measures in this study.

\begin{tabular}{|c|c|c|c|c|c|c|c|c|c|}
\hline & $\begin{array}{l}\text { Coherence } \\
\text { (AAI) }\end{array}$ & $\begin{array}{c}\text { Dismissingscore } \\
\text { (AAI) }\end{array}$ & $\begin{array}{c}\text { Preoccupiedscore } \\
\text { (AAI) }\end{array}$ & 1 & 2 & 3 & 4 & 5 & 6 \\
\hline PAT-RS Security & $.37 *$ & $-.37 *$ & .12 & $-.70 * *$ & -.32 & $.60 * *$ & $.51 * *$ & $-.59 * *$ & .12 \\
\hline 1. PAT-RS Deactivation & $-.53 *$ & $.50 * *$ & -.21 & - & -.35 & $-.53 * *$ & $-.56^{* *}$ & $.76 * *$ & -.24 \\
\hline 2. PAT-RS Hyperactivation & .17 & -.21 & -.06 & - & - & -.02 & .05 & -.25 & .21 \\
\hline 3. Coherence (PRI-T) & $.77 * *$ & $-.65 * *$ & -.07 & - & - & - & $.58 * *$ & $-.66 * *$ & .19 \\
\hline 4. PACS security & $.86^{* *}$ & $-.47 * *$ & $-.45^{*}$ & - & - & - & - & $-.58 * *$ & $-.40^{*}$ \\
\hline 5. PACS avoidance & $-.53 * *$ & $.62 * *$ & -.30 & - & - & - & - & - & $-.42 *$ \\
\hline 6. PACS resistance & -.23 & -.34 & $.79 * *$ & - & - & - & - & - & - \\
\hline
\end{tabular}

AAI, Adult Attachment Interview; PAT-RS, Patient Attachment to Therapist Rating Scale; PRI-T, Patient Relationship Interview at Termination; PACS, Patient Attachment Coding System. *Correlation is significant at $\mathrm{P}<.05$; ** correlation is significant at $\mathrm{P}<.01$.

Table 4. Adult Attachment Interview (AAI) vs Patient Attachment Coding System (PACS) classification (Patient Relationship Interview at Termination, PRI-T): 3-way comparisons.

\begin{tabular}{llccccc}
\hline & \multicolumn{2}{c}{ Classification of the AAI (three-way) } \\
\hline & & Dismissing & Secure & Preoccupied & Total \\
\hline PACS classification (PRI-T) & A & 7 & 0 & 0 & 7 & 17 \\
\hline & B & 2 & 15 & 0 & 6 \\
\hline & C & 1 & 0 & 15 & 5 & 30 \\
\hline
\end{tabular}

$\kappa=.84 ; \mathrm{P}<.001$. 
example by ambivalence, as previously found by Talia and colleagues (2014). On a different note, the lack of significant associations between the AAI Preoccupied score and patients' Coherence in the PRI-T may suggest that some of the discourse markers that are linked to lower coherence in the AAIs of preoccupied patients (for example, the overinvolved and angry discussions of past relationships with parents) may not be especially salient in a discussion of the patient-therapist relationship in a brief treatment.

The finding that the PACS can be applied reliably and validly to a post-treatment interview merits discussion. Up until the present study, the PACS has been used only for coding psychotherapy transcripts, which left it unclear whether the communication markers assessed by the PACS arise only in the context of treatment or if they reflect more general communicative tendencies. This study suggests that the PACS markers can be reliably identified in conversation with a stranger (i.e. the interviewer) and are thus not likely to be specific to the psychotherapy context. If this is the case, avoidant patients' tendency to share sparse descriptions of experience or preoccupied patients' tendency to provide exaggerated or confusing accounts of situations might arise in other sorts of interactions as well. Thus, future research should investigate further the extent to which PACS captures a trait-like interpersonal characteristic present in individuals' interactions with others outside of psychotherapy.

Some interesting clinical implications emerge from our study's results. Safran believed that a central ingredient of relational treatment is for the patient to attend to experiences in the therapeutic relationship as sources of new learning and reflect back on such experiences, perhaps in times of distress, outside of sessions (Safran \& Muran, 2000). Our results seem to suggest that not all patients come to therapy similarly equipped for this process. In this study, we found that a patient's pre-treatment characteristics (i.e. patients' AAI status) influenced how the patient remembered and reflected upon the therapeutic relationship in an interview. According to our results, patients who are more dismissing at intake may be less likely to recall specific memories from treatment after treatment ends. Patients rated as more preoccupied may tend to share narratives about the therapeutic relationship that are incoherent and unbalanced, which could suggest an incomplete or one-sided representation of their experience of the therapist. Thus, therapists working with dismissing and preoccupied patients might need to provide more explicit scaffolding in sessions in order to help these patients attend to and reflect upon the therapeutic relationship in a substantive way.

The high association between patients' pre-treatment AAI and our three post-treatment measures indicates that there may be more continuity than change in the way patients speak about relationships in the two interviews. This could be taken to suggest that, at least in this sample, pa- tients' AAI-status remained relatively stable over the course of treatment - perhaps because changes in patients' attachment status take longer than 30 sessions. Such conclusion would be in line with recent evidence (Daniel et al., 2016) that suggests that improvement in patients' security may not be as common as studies on patients with severe psychopathology had previously suggested (e.g., Levy et al., 2006; Stovall-McClough \& Cloitre, 2003; Tmej et al., 2018). It must be noted, however, that we did not collect a baseline measure of any of the instruments that we applied to the PRI-T, and thus our study does not allow to draw any firm conclusions regarding changes in attachment status in the patients of our sample.

At the time in which this study was conceived, Safran was interested in eventually assessing patients' narratives during the PRI-T as a possible measure of treatment outcome. In carrying this idea forward, future studies should administer and code the PRI-T at the beginning of treatment and compare ratings with those obtained at the end in order to assess whether any changes in the experience, representation, and communications about the therapeutic relationship occurred over the course of treatment. These studies could shed light on whether change in these attachment-related capacities is possible, as well as studying the relationship between such changes and treatment outcome. Further research should also explore in more depth the relationships between the measures examined in this study, each of which seem to tap into a different aspect of attachment. For example, it could be that greater security in the therapeutic relationship enables patients to speak more coherently about the therapist in an interview. Alternately, it may be that patients' generalized capacity to communicate openly about their experience is what facilitates more secure experiences with the therapist and the relaying of coherent narratives about the therapeutic relationship to an interviewer. While we were not able to test these hypotheses due to our small sample size, future adequately powered studies should test if all of the three interview measures included in our study independently contribute to predicting patients' AAI ratings.

This study has a number of significant limitations. First, the current study does not examine data from therapy sessions. Therefore, it is possible that some patients might have attained greater security in their actual interactions with the therapist than what their representation of the therapeutic relationship in the PRI-T indicated. Future studies should investigate the degree to which any changes in the therapeutic relationship or the patients' discourse in sessions carry over to a post-treatment interview. Second, our findings are based on a small sample of relatively high-functioning outpatients, and they may thus not generalize to other clinical populations. This is particularly important as we omitted from our analyses the Unresolved (U) AAI classification, which is more prevalent in high-risk populations (Bakermans-Kranenburg \& van IJzendoorn, 2009) than it was in the current study 
sample. Third, all patients in our sample received BRT, a time-limited treatment with an explicit focus on the therapeutic relationship. Thus, the associations between the AAI and the PRI-T may be greater in this sample than in samples where patients have fewer occasions in-session to reflect upon their relationship with the therapist.

Jeremy Safran approached psychotherapy research with openness to the wide range of questions that could be generated, often more excited by the new questions that would arise than by the answers themselves. His vision for integrating attachment theory into psychotherapy research was motivated by a wish to empirically demonstrate the mutative potential of the therapeutic relationship and its necessary reactivation of patterns established early in development. His interest in the transformative power of the therapeutic relationship resonated with Bowlby's early suggestion that the therapist serves as a secure base from which patients can explore and make sense of early experiences. Although our study only provides preliminary evidence regarding these hypotheses, we hope that it generates fodder for future investigations that will continue to capture Jeremy's spirit of curiosity and openness to the unknown.

\section{References}

Ainsworth, M. S., Blehar, M. C., Waters, E., \& Wall, S. (1978). Patterns of attachment: A psychological study of the strange situation. Hillsdale, NJ: Lawrence Erlbaum.

Allen, J. G., Stein, H., Fonagy, P., Fultz, J., \& Target, M. (2005). Rethinking adult attachment: A study of expert consensus. Bulletin of the Menninger Clinic, 69, 59-80. doi: 10.1521/bumc.69.1.59.62266

Ammaniti, M., Dazzi, N., \& Muscetta, S. (2008). The AAI in a clinical context: Some experiences and illustrations. In $\mathrm{H}$. Steele \& M. Steele (Eds.), Clinical applications of the Adult Attachment Interview (pp. 236-269). New York, NY, US: Guilford Press.

Bakermans-Kranenburg, M. J., \& van IJzendoorn, M. H. (2009). The first 10,000 Adult Attachment Interviews: distributions of adult attachment representations in clinical and non-clinical groups. Attachment \& Human Development, 11(3), 223263. doi: 10.1080/14616730902814762

Bate, J., Talia, A., \& Wyner, R. N. (2012). Coherence of transcript in patient-therapist relationship narrative. Unpublished manuscript, Department of Psychology, The New School for Social Research, New York, NY.

Bowlby, J. (1969/1982). Attachment and loss: Vol 1. Attachment. New York, NY: Basic Books.

Bowlby, J. (1988). A secure base: Clinical applications of attachment theory. London: Routledge.

Bowman, E. A., \& Safran, J. D. (2007). An integrated developmental perspective on insight. In L. G. Castonguay \& C. Hill (Eds.), Insight in Psychotherapy (pp. 401-421). Washington, DC, US: American Psychological Association.

Crowell, J. A., Treboux, D., \& Waters, E. (2002). Stability of attachment representations: The transition to marriage. Developmental Psychology, 38(4), 467. doi: 10.1037/0012-1649. 38.4.467
Daniel, S. I. F., Poulsen, S., \& Lunn, S. (2016). Client attachment in a randomized clinical trial of psychoanalytic and cognitive-behavioral psychotherapy for bulimia nervosa: Outcome moderation and change. Psychotherapy, 53(2), 174. doi: $10.1037 / \mathrm{pst} 0000046$

Diamond, D., Stovall-McClough, C., Clarkin, J., \& Levy, K. (2003). Patient-therapist attachment in the treatment of borderline personality disorder. Bulletin of Menninger Clinic, 67(3), 227-259. Retrieved from http://guilfordjournals. com/loi/bumc

Dykas, M. J., Woodhouse, S. S., Jones, J. D., \& Cassidy, J. (2014). Attachment-related biases in adolescents' memory. Child Development, 85(6), 2185-2201. doi: 10.1111/cdev. 12268

George, C., Kaplan, N., \& Main, M. (1996). Adult Attachment Interview (3rd ed.). Unpublished manuscript, University of California at Berkeley, USA.

Haltigan, J. D., Leerkes, E. M., Wong, M. S., Fortuna, K., Roisman, G. I., Supple, A. J., ... Plamondon, A. (2014). Adult attachment states of mind: Measurement invariance across ethnicity and associations with maternal sensitivity. Child Development, 85(3), 1019-1035. doi: 10.1111/cdev.12180

Haydon, K. C., Roisman, G. I., \& Burt, K. B. (2012). In search of security: The latent structure of the Adult Attachment Interview revisited. Development and Psychopathology, 24(2), 589-606. doi: 10.1017/S0954579412000181

Haydon, K. C., Roisman, G., Marks, M. J., \& Fraley, R. C. (2011). An empirically derived approach to the latent structure of the Adult Attachment Interview: Additional convergent and discriminant validity evidence. Attachment \& Human Development, 13(5), 503-524. doi: 10.1080/146167 34.2011.602253

Hesse, E. (1996). Discourse, memory, and the Adult Attachment Interview: A note with emphasis on the emerging cannot classify category. Infant Mental Health Journal, 17(1), 411. doi: 10.1002/(SICI)1097-0355(199621)17:1\%3C4:: AID-IMHJ1\%3E3.0.CO;2-S

Hesse, E. (1999). The adult attachment interview: Historical and current perspectives. In J. Cassidy \& P. R. Shaver (Eds.), Handbook of Attachment: Theory, research, and clinical applications (pp. 395-433). New York, NY, US: The Guilford Press.

Holmes, J. (2001). The search for a secure base: Attachment theory and psychotherapy. London: Routledge.

Katzow, A. (2011). Keeping a mind in mind: The role of reflective functioning in the process and outcome of Brief Relational Treatment (Doctoral dissertation). New School University.

Kernberg, O. F., Yeomans, F. E., Clarkin, J. F., \& Levy, K. N. (2008). Transference focused psychotherapy: Overview and update. The International Journal of Psychoanalysis, 89(3), 601-620. doi: 10.1111/j.1745-8315.2008.00046.x

Kleinbub, J., Talia, A., Palmieri, A. (2019). Physiological synchronization in the clinical process: a research primer and an attachment-based case study. Manuscript submitted for publication.

Kobak, R. R., Cole, H. E., Ferenz-Gillies, R., \& Fleming, W. S. (1993). Attachment and emotion regulation during motherteen problem solving: A control theory analysis. Child Development, 64(1), 231-245. doi: 10.2307/1131448.

Levy, K. N., Meehan, K. B., Kelly, K. M., Reynoso, J. S., Weber, M., Clarkin, J. F., \& Kernberg, O. F. (2006). Change in attachment patterns and reflective function in a randomized 
control trial of transference-focused psychotherapy for borderline personality disorder. Journal of Consulting and Clinical Psychology, 74(6), 1027-1040. doi: 10.1037/0022006X.74.6.1027

Lilliengren, P., Falkenström, F., Sandell, R., Mothander, P.R., \& Werbart, A. (2015). Secure attachment to therapist, alliance, and outcome in psychoanalytic psychotherapy with young adults. Journal of Counseling Psychology, 62(1), 1-13. doi: $10.1037 / \mathrm{cou} 0000044$

Lilliengren, P., Werbart, A., Mothander, P. R., Ekström, A., Sjögren, S., \& Ögren, M. L. (2014). Patient attachment to therapist rating scale: Development and psychometric properties. Psychotherapy Research, 24(2), 184-201. doi: 10.1080/10503307.2013.867462

Main, M., Goldwyn, M., \& Hesse, E. (2002). Adult Attachment Interview. Scoring manual. Unpublished manuscript. Department of Psychology, Berkeley University.

Main, M., Kaplan, N., \& Cassidy, J. (1985). Security in infancy, childhood, and adulthood: A move to the level of representation. In I. Bretherton \& E. Waters (Eds.), Growing points of attachment theory and research. Monographs of the Society for Research in Child Development (Vol. 50, pp. 66104). Chicago: Chicago University Press. doi: 10.2307/ 3333826

Mallinckrodt, B. (2010). The psychotherapy relationship as attachment: Evidence and implications. Journal of Social and Personal Relationships, 27, 262-270. doi: 10.1177/ 0265407509360905

Mallinckrodt, B., Gantt, D. L., \& Coble, H. M. (1995). Attachment patterns in the psychotherapy relationship. Development of the patient attachment to therapist scale. Journal of Consulting Psychology, 42(3), 307-317. doi: 10.1037/00220167.42.3.307

Mallinckrodt, B., \& Jeong, J. (2015). Meta-analysis of client attachment to therapist: Associations with working alliance and client pretherapy attachment. Psychotherapy, 52(1), 134. doi: $10.1037 / \mathrm{a} 0036890$

Marmarosh, C. L. (2015). Emphasizing the complexity of the relationship: The next decade of attachment-based psychotherapy research. Psychotherapy, 52(1), 12. doi: 10.1037/a0036504

Martin, J., Raby, K. L., Labella, M. H., \& Roisman, G. I. (2017). Childhood abuse and neglect, attachment states of mind, and non-suicidal self-injury. Attachment \& Human Development, 19(5), 425-446. doi: 10.1080/14616734.2017.1330832

Mikulincer, M., Shaver, P., \& Berant, E. (2013). An attachment perspective on therapeutic processes and outcomes. Journal of Personality, 81(6), 606-616. doi: 10.1111/j.14676494.2012.00806.x

Miller-Bottome, M., Talia, A., Eubanks, C., Safran, J. D., \& Muran, J. C. (2019). Secure in-session attachment predicts Rupture Resolution: Negotiating a secure base. Psychoanalytic Psychology, 36(2), 132-138. doi: 10.1037/pap0000232

Miller-Bottome, M., Talia, A., Safran, J. D., \& Muran, J. C. (2018). Resolving alliance ruptures from an attachment-informed perspective. Psychoanalytic Psychology, 35, 175183. doi: $10.1037 /$ pap0000152

Muscetta, S., Dazzi, N., Decoro, A., Ortu, F., \& Speranza, A. M. (1999). "States of mind with respect to attachment" and change in a psychotherapeutic relationship: A study of the coherence of transcript in a short-term psychotherapy with an adolescent. Psychoanalytic Inquiry, 19(5), 885-921. doi: $10.1080 / 07351699909534279$
Parish, M., \& Eagle, M. N. (2003). Attachment to the therapist. Psychoanalytic Psychology, 20(2), 271-286. doi: 10.1037/ 0736-9735.20.2.271

Roisman, G. I., Holland, A., Fortuna, K., Fraley, R. C., Clausell, E., \& Clarke, A. (2007). The adult attachment interview and self-reports of attachment style: An empirical rapprochement. Journal of Personality and Social Psychology, 92, 678-697. doi: 10.1037/0022-3514.92.4.678

Safran, J. D., \& Muran, J. C. (2000). Negotiating the therapeutic alliance: A relational treatment guide. New York, NY: Guilford Press.

Safran, J. D., \& Muran, J. C. (2008). Patient Relationship Interview - Termination. Unpublished manuscript. New School for Social Research.

Safran, J. D., Muran, J. C., \& Samstag, L. W. (1994). Resolving therapeutic alliance ruptures: A task analytic investigation. In A. O. Horvath \& L. S. Greenberg (Eds.), The working alliance: Theory, research, and practice (pp. 225-255). New York, NY: Wiley.

Samstag, L. W., Muran, J. C., Wachtel, P. L., Slade, A., Safran, J. D., \& Winston, A. (2008). Evaluating negative process: A comparison of working alliance, interpersonal behavior, and narrative coherency among three psychotherapy outcome conditions. American Journal of Psychotherapy, 62(2), 165-194. doi: 10.1176/appi.psychotherapy.2008. 62.2 .165

Slade, A. (2005). Parental reflective functioning: An introduction. Attachment \& Human Development, 7(3), 269-281. doi: 10.1080/14616730500245906

Slade, A. (2016). Attachment and adult psychotherapy: Theory, research, and practice. In J. Cassidy \& P. R. Shaver (Eds.), Handbook of attachment: Theory, research, and clinical applications (3rd ed., pp. 131-157). New York, NY: Guilford Press.

Stovall-McClough, K. C., \& Cloitre, M. (2003). Reorganization of unresolved childhood traumatic memories following exposure therapy. Annals of the New York Academy of Sciences, 1008(1), 297-299. doi: 10.1196/annals.1301.036

Talia, A., Daniel, S. I., Miller-Bottome, M., Brambilla, D., Miccoli, D., Safran, J. D., \& Lingiardi, V. (2014). AAI predicts patients' in-session interpersonal behavior and discourse: a "move to the level of the relation" for attachment-informed psychotherapy research. Attachment \& Human Development, 16(2), 192-209. doi: 10.1080/ 14616734.2013 .859161

Talia, A., \& Miller-Bottome, M. (2015). Patient Attachment Coding System 2.0. Unpublished manuscript, Institute of Psychosocial Prevention, Heidelberg University.

Talia, A., Miller-Bottome, M., \& Daniel, S. I. (2017). Assessing attachment in psychotherapy: validation of the patient attachment coding system (PACS). Clinical Psychology \& Psychotherapy, 24(1), 149-161. doi: 10. 1002/cpp.1990

Talia, A., Miller-Bottome, M., Katznelson, H., $\quad$ Pedersen, S. H., Steele, H., Schröder, P., ... Lingiardi, V. (2018). Mentalizing in the presence of another: Measuring reflective functioning and attachment in the therapy process. Psychotherapy Research, 1-14. doi: 10.1080/10503307.2017.1417651

Talia, A., Muzi, L., Lingiardi, V., \& Taubner, S. (2018). How to be a secure base: Therapists' attachment representations and their link to attunement in psychotherapy. Attachment \& Human Development. Advance online publication. doi: 10.1080/14616734.2018.1534247

Tarabulsy, G. M., Larose, S., Bernier, A., Trottier-Sylvain, K., 
Girard, D., Vargas, M., \& Noël, C. (2012). Attachment states of mind in late adolescence and the quality and course of romantic relationships in adulthood. Attachment \& Human Development, 14(6), 621-643. doi: 10.1080/14616734. 2012.728358

Tmej, A., Fischer-Kern, M., Doering, S., Alexopoulos, J., \& Buchheim, A. (2018). Changes in attachment representation in psychotherapy: Is reflective functioning the crucial factor? Zeitschrift für Psychosomatische Medizin und Psychotherapie, 64(3), 222-236. doi: 10.13109/zptm.2018.64. 3.222

Verhage, M. L., Schuengel, C., Madigan, S., Fearon, R. M. P., Oosterman, M., Cassibba, R., ... van IJzendoorn, M. H. (2016). Narrowing the transmission gap: A synthesis of three decades of research on intergenerational transmission of at- tachment. Psychological Bulletin, 2(4), 337-366. doi: 10.1037/bul0000038

Wallin, D. (2007). Attachment and Psychotherapy. New York, NY: Guilford Press.

Waters, E., Treboux, D., Fyffe, C., \& Crowell, J. A. (2005). Adult attachment patterns as continuous variables: Discriminant analysis using AAI state of mind scales. Unpublished manuscript. Department of Psychology, State University of New York.

Zeanah, C. H., Benoit, D., Barton, M. L., \& Hirshberg, L. (1996). Working model of the child interview coding manual. Unpublished manuscript. New Orleans: Division of Infant, Child and Adolescent Psychiatry, Louisiana State University School of Medicine. 